\title{
Reflexive families of closed sets
}

\author{
by \\ Zhongqiang Yang (Shantou) and Dongsheng Zhao (Singapore)
}

\begin{abstract}
Let $S(X)$ denote the set of all closed subsets of a topological space $X$, and $C(X)$ the set of all continuous mappings $f: X \rightarrow X$. A family $\mathcal{A} \subseteq S(X)$ is called reflexive if there exists $\mathcal{F} \subseteq C(X)$ such that $\mathcal{A}=\{A \in S(X): f(A) \subseteq A$ for every $f \in \mathcal{F}\}$. We investigate conditions ensuring that a family of closed subsets is reflexive.
\end{abstract}

Recall [3] that a collection $\mathcal{A}$ of closed subspaces of a Hilbert space $H$ is called reflexive if there exists a collection $\mathcal{F}$ of continuous operators on $H$ such that

$$
\mathcal{A}=\operatorname{Lat}(\mathcal{F})=\{A: A \text { is a closed subspace of } H \text { with } T(A) \subseteq A, \forall T \in \mathcal{F}\} .
$$

Reflexive families of continuous operators are defined in a dual way. See [2-7] for characterizations of such families. In [8], the second author considered reflexive families in concrete categories. For the category SET of sets, he obtained complete characterizations for both reflexive families of sets and reflexive families of mappings. In the present paper we investigate reflexive families in the context of topological spaces.

Given a topological space $X$, let $S(X)$ be the set of all closed subsets of $X$ and $C(X)$ be the set of all continuous mappings $f: X \rightarrow X$. For any $\mathcal{A} \subseteq S(X)$ and $\mathcal{F} \subseteq C(X)$ define

$$
\begin{aligned}
& \operatorname{Alg}(\mathcal{A})=\{f \in C(X): f(A) \subseteq A \text { for every } A \in \mathcal{A}\}, \\
& \operatorname{Lat}(\mathcal{F})=\{A \in S(X): f(A) \subseteq A \text { for every } f \in \mathcal{F}\} .
\end{aligned}
$$

The two mappings $\mathrm{Alg}$ and Lat form a Galois connection between the sets of all subsets of $S(X)$ and $C(X)$, respectively. Thus, for any $\mathcal{A} \subseteq S(X)$ and $\mathcal{F} \subseteq C(X)$ we have

2000 Mathematics Subject Classification: 47A15, 54C05, 54D05, 54E45.

Key words and phrases: reflexive family of closed sets, s-reflexive topological space, strongly zero-dimensional metric space, hereditarily disconnected space, reflexive closed set.

The first author was supported by National Natural Science Foundation of China (No. 10471084) and by Guangdong Provincial Natural Science Foundation (No. 04010985). 
(i) $\operatorname{Lat}(\operatorname{Alg}(\mathcal{A})) \supseteq \mathcal{A}, \operatorname{Alg}(\operatorname{Lat}(\mathcal{F})) \supseteq \mathcal{F}$;

(ii) $\operatorname{Alg}(\operatorname{Lat}(\operatorname{Alg}(\mathcal{A})))=\operatorname{Alg}(\mathcal{A}), \operatorname{Lat}(\operatorname{Alg}(\operatorname{Lat}(\mathcal{F})))=\operatorname{Lat}(\mathcal{F})$.

A family $\mathcal{A} \subseteq S(X)$ is called reflexive if $\mathcal{A}=\operatorname{Lat}(\operatorname{Alg}(\mathcal{A})$ ). Similarly, $\mathcal{F} \subseteq C(X)$ is reflexive if $\mathcal{F}=\operatorname{Alg}(\operatorname{Lat}(\mathcal{F}))$.

As in the general case [8], $\mathcal{A} \subseteq S(X)$ is reflexive if and only if there exists $\mathcal{F} \subseteq C(X)$ such that $\mathcal{A}=\operatorname{Lat}(\mathcal{F})$. Also by (i), $\mathcal{A}$ is reflexive if and only if

$$
\operatorname{Lat}(\operatorname{Alg}(\mathcal{A})) \subseteq \mathcal{A} .
$$

Lemma 1. If $\mathcal{A} \subseteq S(X)$ is reflexive, then:

(a) $X, \emptyset \in \mathcal{A}$.

(b) $\mathcal{B} \subseteq \mathcal{A}$ implies $\bigcap \mathcal{B} \in \mathcal{A}$.

(c) $\mathcal{B} \subseteq \mathcal{A}$ implies $\operatorname{cl}(\bigcup \mathcal{B}) \in \mathcal{A}$.

(d) If $D$ is a connected component of $A \in \mathcal{A}$ and $B \subseteq D$ for some nonempty $B$ in $\mathcal{A}$, then $D \in \mathcal{A}$.

Proof. Only (d) needs verification. For any $f \in \operatorname{Alg}(\mathcal{A}), f(B) \subseteq B$ since $B \in \mathcal{A}$. Also $f(B) \subseteq f(D) \subseteq f(A) \subseteq A$, and $f(D) \cap D \supseteq f(B) \cap B$ $=f(B) \neq \emptyset$. Thus $f(D)$ is a connected set contained in $A$ and has nonempty intersection with the connected component $D$ of $A$, hence $f(D) \subseteq D$. Therefore $D \in \operatorname{Lat}(\operatorname{Alg}(\mathcal{A}))=\mathcal{A}$.

If $X$ is a space with the discrete topology, then $S(X)$ is the set of all subsets of $X$ and $C(X)$ is the set of all mappings from $X$ to $X$. By [8, Theorem 1], a family $\mathcal{A}$ of subsets of $X$ is reflexive if and only if $\mathcal{A}$ is closed under arbitrary unions and intersections, so every family $\mathcal{A}$ of closed subsets of a discrete space satisfying conditions (a)-(c) in Lemma 1 is reflexive. A natural question is: besides the discrete topological spaces, what other spaces also have this property?

Definition 1. A topological space $X$ is called s-reflexive if every family $\mathcal{A}$ of closed subsets of $X$ satisfying conditions (a)-(c) in Lemma 1 is reflexive.

The main results in this paper are: every strongly zero-dimensional complete metric space is s-reflexive; every countable metric space is s-reflexive; every Hausdorff s-reflexive space is hereditarily disconnected. From these it is deduced that a locally compact metric space is s-reflexive if and only if it is zero-dimensional.

Lemma 2. For every topological space $X$, the following conditions are equivalent:

(1) For any nonempty proper closed subset $B$ and any finite subset $D$ of $X$, both $B \backslash D$ and $B \cup D$ are closed. 
(2) For any nonempty finite set $D \subseteq X$ and any element $b \in X$, the mapping $f_{D, b}$ which sends $D$ to $b$ and is the identity on $X \backslash D$, is continuous.

(3) For any points $a, b \in X$, the above mapping $f_{\{a\}, b}$ is continuous.

REMARK 1. If $X$ satisfies the equivalent conditions in Lemma 2 with $|X| \neq 2$ and there exists one closed singleton $\{a\}$, then $X$ is $T_{1}$. The assumption that $|X| \neq 2$ is essential.

Let $\mathcal{A} \subseteq S(X)$ be a collection of closed subsets of $X$ satisfying conditions (a)-(c) in Lemma 1. For each $Y \in S(X)$, let $\phi_{\mathcal{A}}(Y)=\bigcap\{A \in \mathcal{A}: Y \subseteq A\}$. If no confusion occurs, we simply write $\phi(Y)$ for $\phi_{\mathcal{A}}(Y)$. The following lemma can be verified easily.

Lemma 3. Let $\mathcal{A} \subseteq S(X)$ satisfy conditions (a)-(c).

(1) For any $Y \subseteq X, \phi(Y) \in \mathcal{A}$. And $Y \in \mathcal{A}$ if and only if $Y=\phi(Y)$.

(2) For any $B \in S(X), B \in \mathcal{A}$ if and only if $\phi(\{x\}) \subseteq B$ for all $x \in B$.

(3) For any $B \in S(X), B \in \mathcal{A}$ if and only if $B=\bigcup\{\phi(\{x\}): x \in B\}$.

Lemma 4. Let $\mathcal{A} \subseteq S(X)$ satisfy conditions (a)-(c). For each $U \subseteq X$ define $\kappa(U)=\{x \in X: \phi(\{x\}) \cap U \neq \emptyset\}$. Then

(1) $\kappa(V) \supseteq V$ for all $V \subseteq X$.

(2) $\kappa\left(\bigcup_{i \in I} U_{i}\right)=\bigcup_{i \in I} \kappa\left(U_{i}\right)$.

(3) If $U$ is an open subset of $X$, then $\kappa(U)$ is also open.

Proof. We only prove (3). Let $U$ be an open subset of $X$ and $\left(x_{\lambda}\right)_{\lambda \in D}$ be a net in $X \backslash \kappa(U)$ which converges to a point $a \in X$. For each $\lambda \in D$, $\phi\left(\left\{x_{\lambda}\right\}\right) \cap U=\emptyset$. Thus $\operatorname{cl}\left(\bigcup_{\lambda \in D} \phi\left(\left\{x_{\lambda}\right\}\right)\right) \cap U=\emptyset$. By (c), the set $B=$ $\operatorname{cl}\left(\bigcup_{\lambda \in D} \phi\left(\left\{x_{\lambda}\right\}\right)\right)$ is in $\mathcal{A}$. Now $a \in B$, so $\phi(\{a\}) \subseteq B \subseteq X \backslash U$. Hence $\phi(\{a\}) \cap U=\emptyset$, which implies $a \in X \backslash \kappa(U)$. Therefore, $\kappa(U)$ is open.

TheOrem 1. If a space $X$ satisfies the equivalent conditions in Lemma 2, then $X$ is s-reflexive.

Proof. Suppose $\mathcal{A} \subseteq S(X)$ satisfies (a)-(c). Let $B$ be a closed set not in $\mathcal{A}$. By Lemma $3(2)$, there is $a \in B$ such that $\phi(\{a\}) \nsubseteq \subseteq B$. Choose a point $b \in \phi(\{a\}) \backslash B$. The mapping $f_{\{a\}, b}$ defined in Lemma 2 is continuous. For every $A \in \mathcal{A}$, if $a \in A$ then $\phi(\{a\}) \subseteq A$, so $f(a)=b \in \phi(\{a\}) \subseteq A$, hence $f(A) \subseteq A$. If $a \notin A$, then $f(A)=A$. Thus $f \in \operatorname{Alg}(\mathcal{A})$. But $f(B) \nsubseteq B$, so $\mathcal{A} \supseteq \operatorname{Lat}(\operatorname{Alg}(\mathcal{A}))$ and hence $\mathcal{A}=\operatorname{Lat}(\operatorname{Alg}(\mathcal{A}))$, which shows that $\mathcal{A}$ is reflexive.

EXAMPlE 1. (1) Every discrete and every anti-discrete space satisfies the conditions in Lemma 2 , so they are s-reflexive.

(2) Let $\lambda$ be an infinite cardinal and $X$ be any nonempty set. Define $\tau$ to be the topology consisting of all subsets whose complements are either 
$X$ or have cardinality less than $\lambda$. Then $(X, \tau)$ satisfies the conditions in Lemma 2 , so it is $s$-reflexive.

(3) Suppose $E$ is a subset of a nonempty set $X$. Define $\tau_{E}$ to be the topology consisting of those $A \subseteq X$ such that either $A=X$, or $A \backslash E$ is finite. Then $\left(X, \tau_{E}\right)$ satisfies the conditions in Lemma 2 , and so it is $s$-reflexive.

(4) The Euclidean interval $X=[-1,1]$ is not s-reflexive. As a matter of fact, let $\mathcal{A}=\{A \in S(X): A \subseteq[0,1]$ or $A \ni 1\}$. Then $\mathcal{A}$ satisfies conditions (a)-(c) in Lemma 1 . For every $f \in \operatorname{Alg}(\mathcal{A})$ and every $x \in X$, since $\{x, 1\} \in \mathcal{A}$, we have $f(\{x, 1\}) \subseteq\{x, 1\}$, so either $f(x)=x$ or $f(x)=1$. Let $A=\{x \in[-1,1): f(x)=x\}$ and $B=\{x \in[-1,1): f(x)=1\}$. Then $A$ and $B$ are two disjoint closed sets in $[-1,1)$ and $A \cup B=[-1,1)$. Hence $A=\emptyset$ or $B=\emptyset$ because $[-1,1)$ is connected. For every $x \in[0,1]$, since $\{x\} \in \mathcal{A}$ we have $f(x)=x$. It follows that $A \supseteq[0,1) \neq \emptyset$. This implies $A=[-1,1)$. Note that $f(1)=1$ for every $f \in \operatorname{Alg}(\mathcal{A})$ because $\{1\} \in \mathcal{A}$. Thus $\operatorname{Alg}(\mathcal{A})=\left\{\operatorname{id}_{X}\right\}$. But $\operatorname{Lat}\left(\left\{\operatorname{id}_{X}\right\}\right)=S(X) \neq \mathcal{A}$. Hence $\mathcal{A}$ is not reflexive.

Since the interval $[-1,1]$ equipped with the discrete topology is s-reflexive and the Euclidean interval is a continuous image of it, it follows that continuous images of s-reflexive spaces need not be $s$-reflexive.

Let $\mathcal{U}$ be an open cover of a strongly zero-dimensional metric space $X$ and $\varepsilon>0$. Then there exists a locally finite open refinement $\mathcal{W}$ of $\mathcal{U}$ such that for every $W \in \mathcal{V}$, the diameter $\operatorname{diam}(W)$ of $W$ is less than $\varepsilon$. It then follows from [1, Theorems 7.3.2 and 7.2.4] that there exists a refinement $\mathcal{V}$ of $\mathcal{W}$ consisting of pairwise disjoint clopen sets. Thus we have the following lemma.

Lemma 5. Let $\mathcal{U}$ be an open cover of a strongly zero-dimensional metric space $X$. Then for any $\varepsilon>0$, there is a refinement $\mathcal{V}$ of $\mathcal{U}$ such that $\mathcal{V}$ consists of pairwise disjoint clopen sets and $\operatorname{diam}(V)<\varepsilon$ for all $V \in \mathcal{V}$.

THEOREM 2. Every strongly zero-dimensional complete metric space is s-reflexive.

Proof. Let $(X, d)$ be a strongly zero-dimensional complete metric space and let $\mathcal{A}$ be a family of closed sets in $X$ satisfying conditions (a)-(c) in Lemma 1. Suppose $B \in S(X)$ and $B \notin \mathcal{A}$. We shall define an $f \in \operatorname{Alg}(\mathcal{A})$ so that $f(B) \nsubseteq B$. By Lemma 3(2), there is $b \in B$ such that $\phi(\{b\}) \nsubseteq B$. Choose $c \in \phi(\{b\}) \backslash B$ and a clopen set $U_{0}$ with $\operatorname{diam}\left(U_{0}\right)<1$ and $c \in U_{0} \subseteq$ $X \backslash B$. By Lemma $4(3), \kappa\left(U_{0}\right)$ is open and $b \in \kappa\left(U_{0}\right)$, there is a clopen set $V_{0}$ such that $\operatorname{diam}\left(V_{0}\right)<1, b \in V_{0} \subseteq \kappa\left(U_{0}\right)$ and $V_{0} \cap U_{0}=\emptyset$.

Now we construct two sequences $\left\{\mathcal{U}_{n}\right\}_{n=1}^{\infty}$ and $\left\{\mathcal{V}_{n}\right\}_{n=1}^{\infty}$ of collections of pairwise disjoint nonempty clopen sets, and a mapping $\alpha_{n}: \mathcal{V}_{n} \rightarrow \mathcal{U}_{n}$ for each $n$, such that the following conditions are satisfied: 
(i) $\bigcup \mathcal{U}_{n}=U_{0}, \bigcup \mathcal{V}_{n}=V_{0}$ for each $n$;

(ii) $\operatorname{diam}(U) \leq 1 / n$ for every $U \in \mathcal{U}_{n}$;

(iii) $\mathcal{U}_{n+1}$ is a refinement of $\mathcal{U}_{n}$, and $\mathcal{V}_{n+1}$ is a refinement of $\mathcal{V}_{n}$;

(iv) $V \subseteq \kappa\left(\alpha_{n}(V)\right)$ for every $V \in \mathcal{V}_{n}$;

(v) if $m \leq n$ and $V \in \mathcal{V}_{m}, W \in \mathcal{V}_{n}$ then either $W \subseteq V$ or $V \cap W=\emptyset$;

(vi) if $m \leq n, W \in \mathcal{V}_{n}, V \in \mathcal{V}_{m}$ and $W \subseteq V$, then $\alpha_{n}(W) \subseteq \alpha_{m}(V)$.

First, let $\mathcal{U}_{1}=\left\{U_{0}\right\}, \mathcal{V}_{1}=\left\{V_{0}\right\}$ and $\alpha_{1}\left(V_{0}\right)=U_{0}$. Then the above six conditions are satisfied.

Now suppose for each $i \leq k, \mathcal{U}_{i}, \mathcal{V}_{i}$ and $\alpha_{i}: \mathcal{V}_{i} \rightarrow \mathcal{U}_{i}$ have been defined and satisfy (i)-(vi). For any $U \in \mathcal{U}_{k}$, by Lemma 5 there exists a family $\mathcal{U}_{k+1}(U)$ of pairwise disjoint clopen sets in $X$ such that $\bigcup \mathcal{U}_{k+1}(U)=U$ and $\operatorname{diam}(W) \leq 1 /(k+1)$ for every $W \in \mathcal{U}_{k+1}(U)$. Let $\mathcal{U}_{k+1}=\bigcup\left\{\mathcal{U}_{k+1}(U)\right.$ : $\left.U \in \mathcal{U}_{k}\right\}$. Obviously $\mathcal{U}_{k+1}$ is a refinement of $\mathcal{U}_{k}$. Next, for each $V \in \mathcal{V}_{k}$, by (iv) we have $V \subseteq \kappa\left(\alpha_{k}(V)\right)$. Note that $\kappa$ preserves unions by Lemma 4(2); it follows that $\left\{\kappa(W) \cap V: W \in \mathcal{U}_{k+1}\left(\alpha_{k}(V)\right)\right\}$ is an open cover of $V$. Again, by Lemma 5 , there is a cover $\mathcal{V}_{k+1}(V)$ of $V$ consisting of pairwise disjoint clopen sets and $\mathcal{V}_{k+1}(V)$ is finer than $\left\{\kappa(W) \cap V: W \in \mathcal{U}_{k+1}\left(\alpha_{k}(V)\right)\right\}$. Put $\mathcal{V}_{k+1}=\bigcup\left\{\mathcal{V}_{k+1}(V): V \in \mathcal{V}_{k}\right\}$. Then $\mathcal{V}_{k+1}$ is a refinement of $\mathcal{V}_{k}$.

To define $\alpha_{k+1}$, for each $A \in \mathcal{V}_{k+1}$, there is a unique $V \in \mathcal{V}_{k}$ such that $A \in \mathcal{V}_{k+1}(V)$. Then $A \subseteq \kappa(E) \cap V$ for some $E \in \mathcal{U}_{k+1}\left(\alpha_{k}(V)\right) \subseteq \mathcal{U}_{k+1}$. Such a set $E$ need not be unique. Choose any of them and let $\alpha_{k+1}(A)=E$. Thus we have defined a mapping $\alpha_{k+1}: \mathcal{V}_{k+1} \rightarrow \mathcal{U}_{k+1}$.

Conditions (i)-(iv) for $k+1$ follow immediately from the construction of these objects. To show (v) for $k+1$, let $m \leq k+1$; then $\mathcal{V}_{k+1}$ is a refinement of $\mathcal{V}_{m}$. If $V \in \mathcal{V}_{m}, W \in \mathcal{V}_{k+1}$ and $W \nsubseteq V$, then $W \subseteq V^{\prime}$ for some $V^{\prime} \in \mathcal{V}_{m}$, where $V^{\prime} \neq V$, thus $W \cap V \subseteq V^{\prime} \cap V=\emptyset$. To prove (vi) it is enough to check the case where $m=k$ and $n=k+1$. Let $V \in \mathcal{V}_{k}, W \in \mathcal{V}_{k+1}$ and $W \subseteq V$. By the definition of $\alpha_{k+1}, \alpha_{k+1}(W)=E$ for some $E \in \mathcal{U}_{k+1}\left(\alpha_{k}(V)\right)$, hence $\alpha_{k+1}(W)=E \subseteq \alpha_{k}(V)$.

By induction we have defined the sequences $\left\{\mathcal{U}_{n}\right\}_{n=1}^{\infty},\left\{\mathcal{V}_{n}\right\}_{n=1}^{\infty}$, and the mapping $\alpha_{n}$ for each $n$.

Now define the mapping $f: X \rightarrow X$ as follows:

$$
\{f(x)\}= \begin{cases}\{x\} & \text { if } x \in X \backslash V_{0}, \\ \bigcap_{n=1}^{\infty}\left\{\alpha_{n}\left(V_{n}\right): x \in V_{n} \in \mathcal{V}_{n}\right\} & \text { if } x \in V_{0} .\end{cases}
$$

First, $f$ is well defined. As a matter of fact, if $x \in V_{0}$, then for each $n$, there is a unique $V_{n} \in \mathcal{V}_{n}$ with $x \in V_{n}$. If $x \in V_{n} \in \mathcal{V}_{n}, x \in V_{m} \in \mathcal{V}_{m}$ and $m \leq n$, then it follows from (v) and (vi) that $\emptyset \neq \alpha_{n}\left(V_{n}\right) \subseteq \alpha_{m}\left(V_{m}\right)$. Thus $\left\{\alpha_{n}\left(V_{n}\right): n \in \mathbb{N}\right\}$ is a sequence of closed sets whose every finite subfamily has a nonempty intersection. Furthermore, $\operatorname{diam}\left(\alpha_{n}\left(V_{n}\right)\right) \leq 1 / n$ for each $n$ and $X$ is complete, so the set $\bigcap_{n=1}^{\infty} \alpha_{n}\left(V_{n}\right)$ is a singleton. 
The mapping $f$ is clearly continuous on $X \backslash V_{0}$. For any $x \in V_{0}$ and every $\varepsilon>0$, there exists $V_{n}$ such that $x \in V_{n} \in \mathcal{V}_{n}$ and $\alpha_{n}\left(V_{n}\right) \subseteq B(f(x), \varepsilon)$. For each $y \in V_{n}$, by the definition of $f, f(y) \in \alpha_{n}\left(V_{n}\right)$, hence $f(y) \in B(f(x), \varepsilon)$. This shows that $f$ is also continuous on $X \backslash V_{0}$.

For any $x \in X$, we show $f(x) \in \phi(\{x\})$. If $x \notin V_{0}, f(x)=x \in \phi(\{x\})$. If $x \in V_{0}$, then for each $n$, there is a unique $V_{n} \in \mathcal{V}_{n}$ such that $x \in V_{n}$. Moreover, $V_{n} \subseteq \kappa\left(\alpha_{n}\left(V_{n}\right)\right)$, so $\phi(\{x\}) \cap \alpha_{n}\left(V_{n}\right) \neq \emptyset$. Choose any $x_{n} \in$ $\phi(\{x\}) \cap \alpha_{n}\left(V_{n}\right)$. Then the sequence $\left\{x_{n}\right\}$ converges to $f(x)$, so $f(x) \in \phi(\{x\})$ because $\phi(\{x\})$ is closed.

Now for each $A \in \mathcal{A}$, if $x \in A$ and $x \in X \backslash V_{0}$, then $f(x)=x \in A$; if $x \in A \cap V_{0}$, then $f(x) \in \phi(\{x\}) \subseteq A$, so again $f(x) \in A$. Thus $f \in \operatorname{Alg}(\mathcal{A})$. However, $b \in V_{0} \cap B, f(b) \in \alpha_{1}\left(V_{0}\right)=U_{0}$, and $U_{0} \cap B=\emptyset$, so $f(b) \notin B$. Hence $f(B) \nsubseteq B$, which implies $B \notin \operatorname{Lat}(\operatorname{Alg}(\mathcal{A}))$. The proof is complete.

Note that in constructing the sequences $\left\{\mathcal{U}_{n}\right\}_{n=1}^{\infty},\left\{\mathcal{V}_{n}\right\}_{n=1}^{\infty}$ and mappings $\alpha_{n}$ we did not make use of the completeness of $X$.

By [1, Corollary 6.2.8] every countable metric space is strongly zerodimensional but is not necessarily complete.

TheOREM 3. Every countable metric space is s-reflexive.

Proof. Let $X$ be a countable metric space. Then $X$ is strongly zerodimensional. We show that $X$ is $s$-reflexive. The proof is similar to that of Theorem 2. Again, let $\mathcal{A}$ be a family of closed sets in $X$ satisfying (a)(c) in Lemma 1 , and $B \notin \mathcal{A}$. Let $b \in B$ such that $\phi(\{b\}) \nsubseteq B$. Choose $c \in \phi(\{b\}) \backslash B$ and a clopen set $U_{0}$ with $\operatorname{diam}\left(U_{0}\right)<1$ and $c \in U_{0} \subseteq X \backslash B$. Since $\kappa\left(U_{0}\right)$ is open and $b \in \kappa\left(U_{0}\right)$ and $b \notin U_{0}$, there is a clopen set $V_{0}$ such that $\operatorname{diam}\left(V_{0}\right)<1, b \in V_{0} \subseteq \kappa\left(U_{0}\right)$ and $V_{0} \cap U_{0}=\emptyset$.

In the following we assume that $V_{0}$ is an infinite set. If $V_{0}$ has only $n$ elements, we stop our inductive constructions in the $n$th step. The rest of the arguments will be the same as in the infinite case.

Arrange $V_{0}$ as $\left\{x_{1}, x_{2}, \ldots\right\}$, where $x_{1}=b$.

We now define by induction two sequences $\left\{\mathcal{U}_{n}\right\}_{n=1}^{\infty}$ and $\left\{\mathcal{V}_{n}\right\}_{n=1}^{\infty}$ of pairwise disjoint clopen sets of $X$, a map $\alpha_{n}: \mathcal{V}_{n} \rightarrow \mathcal{U}_{n}$ for each $n$, and $f\left(x_{n}\right)(n \in \mathbb{N})$, such that conditions (i)-(vi) in the proof of Theorem 2 hold and moreover:

(vii) for any $1 \leq i<j \leq n$, if $x_{i} \in V \in \mathcal{V}_{n}$ and $x_{j} \in V^{\prime} \in \mathcal{V}_{n}$, then $V \cap V^{\prime}=\emptyset$

(viii) if $i \leq n$ and $x_{i} \in V \in \mathcal{V}_{n}$, then $f\left(x_{i}\right) \in \alpha_{n}(V) \cap \phi\left(x_{i}\right)$.

For $n=1$ we let $\mathcal{U}_{1}=\left\{U_{0}\right\}, \mathcal{V}_{1}=\left\{V_{0}\right\}, \alpha_{1}\left(V_{0}\right)=U_{0}$, and $f\left(x_{1}\right)=c$.

Suppose for each $i \leq n, \mathcal{U}_{i}, \mathcal{V}_{i}, \alpha_{i}$ and $f\left(x_{i}\right)$ have been defined and they satisfy the required conditions. To define these objects for $n+1$, for each $U \in \mathcal{U}_{n}$ choose $\mathcal{U}_{n+1}(U)$ as a collection of pairwise disjoint clopen sets with 
diameter less than $1 /(n+1)$ and $\bigcup \mathcal{U}_{n+1}(U)=U$. Put $\mathcal{U}_{n+1}=\bigcup\left\{\mathcal{U}_{n+1}(U)\right.$ : $\left.U \in \mathcal{U}_{n}\right\}$. Let $V \in \mathcal{V}_{n}$. We consider three cases:

CAsE A: $V \cap\left\{x_{1}, \ldots, x_{n}, x_{n+1}\right\}=\emptyset$ or $V \cap\left\{x_{1}, \ldots, x_{n}, x_{n+1}\right\}=\left\{x_{n+1}\right\}$. Then $\mathcal{V}_{n+1}(V)$ and the restriction of $\alpha_{n+1}$ to $\mathcal{V}_{n+1}(V)$ are defined as in the proof of Theorem 2. When the intersection is $\left\{x_{n+1}\right\}$, there is $W \in \mathcal{V}_{n+1}(V)$ with $x_{n+1} \in W$. Then define $f\left(x_{n+1}\right)$ to be any point in $\alpha_{n+1}(W) \cap \phi\left(x_{n+1}\right)$. Note that as $x_{n+1} \in W \subseteq \kappa\left(\alpha_{n+1}(W)\right)$, such a point exists.

CASE B: $V \cap\left\{x_{1}, \ldots, x_{n}, x_{n+1}\right\}=\left\{x_{i}, x_{n+1}\right\}$ for some $i \leq n$. Choose two disjoint clopen sets $C$ and $D$ such that $x_{i} \in C, x_{n+1} \in D$, and $C \subseteq \kappa\left(U^{\prime}\right) \cap V$ for some $U^{\prime} \in \mathcal{U}_{n+1}\left(\alpha_{n}(V)\right)$ with $f\left(x_{i}\right) \in U^{\prime}$ (note: by induction assumption $x_{i} \in V \in \mathcal{V}_{n}$ implies $f\left(x_{i}\right) \in \alpha_{n}(V) \cap \phi\left(x_{i}\right)$, so there is $U^{\prime} \in \mathcal{U}_{n+1}\left(\alpha_{n}(V)\right)$ with $f\left(x_{i}\right) \in U^{\prime} \cap \phi\left(x_{i}\right)$; it follows that $U^{\prime} \cap \phi\left(x_{i}\right) \neq \emptyset$ and hence $\left.x_{i} \in \kappa\left(U^{\prime}\right)\right)$. Also $D \subseteq \kappa\left(W^{\prime}\right) \cap V$ for some $W^{\prime} \in \mathcal{U}_{n+1}\left(\alpha_{n}(V)\right)$. Now choose a refinement $\mathcal{V}_{n+1}(V)$ of $\left\{\kappa(W) \cap V: W \in \mathcal{U}_{n+1}\left(\alpha_{n}(V)\right)\right\}$ consisting of pairwise disjoint clopen sets and $\mathcal{V}_{n+1}(V)$ contains both $C$ and $D$ as members. Define $\alpha_{n+1}$ on $\mathcal{V}_{n+1}(V)$ by letting $\alpha_{n+1}(C)=U^{\prime}$, and $\alpha_{n+1}(F)$ as before if $F \neq C$. Also define $f\left(x_{n+1}\right)$ to be any point in $\alpha_{n+1}(D) \cap \phi\left(x_{n+1}\right)$ (note: $\alpha_{n+1}(D) \cap$ $\phi\left(x_{n+1}\right) \neq \emptyset$ because $\left.x_{n+1} \in D \subseteq \kappa\left(\alpha_{n+1}(D)\right)\right)$.

CAsE C: $V \cap\left\{x_{1}, \ldots, x_{n}, x_{n+1}\right\}=\left\{x_{i}\right\}$ for some $i \leq n$. Then $\mathcal{V}_{n+1}(V)$ is defined as in the proof of Theorem 2 .

Finally, let $\mathcal{V}_{n+1}=\bigcup\left\{\mathcal{V}_{n+1}(V): V \in \mathcal{V}_{n}\right\}$. Since there is a unique $V \in \mathcal{V}_{n}$ that contains $x_{n+1}$ and satisfies the condition in either Case A or Case B, $f\left(x_{n+1}\right)$ is defined.

Let $g: X \rightarrow X$ be defined by $g(x)=x$ for $x \notin V_{0}$ and $g\left(x_{i}\right)=f\left(x_{i}\right)(i=$ $1,2, \ldots)$. From the above construction it is clear that $g(x) \in \phi(x)$ for all $x \in X$, thus $g(A) \subseteq A$ for all $A \in \mathcal{A}$. In addition $g(b)=f(b)=c \notin B$, so $g(B) \nsubseteq B$. To complete the proof we only need to verify that $g$ is continuous, and for this it is enough to show that $f$ is continuous on $V_{0}$. For any $x=x_{n} \in V_{0}$ and any $\varepsilon>0$, choose $m \geq n$ with $1 / m<\varepsilon$. Let $x_{n} \in V \in \mathcal{V}_{m}$. Then $f\left(x_{n}\right) \in \alpha_{m}(V)$. If $i \geq m$ and $x_{i} \in V$, then there is $W \in \mathcal{V}_{i}$ with $x_{i} \in W \subseteq V$. Then $f\left(x_{i}\right) \in \alpha_{i}(W) \subseteq \alpha_{m}(V)$. Thus $d\left(f\left(x_{n}\right), f\left(x_{i}\right)\right)<\operatorname{diam}(V)<1 / m<\varepsilon$. Hence $f$ is continuous at $x_{n}$.

A space is called hereditarily disconnected if it does not contain any connected subset of cardinality larger than one.

LEMMA 6. Every $T_{1}$ connected space with more than two elements contains a proper connected subset with more than one element.

LEMMA 7. Every s-reflexive Hausdorff space is hereditarily disconnected.

Proof. Let $X$ be an s-reflexive Hausdorff space. If $X$ has at most two elements, it is clearly hereditarily disconnected. Now assume $X$ has more 
than two elements. Suppose $X$ is not hereditarily disconnected. If $X$ is not connected, then as it is not hereditarily disconnected, one of its connected components, say $B$, is a proper non-singleton connected subset. If $X$ is connected, by Lemma 6 there also exists a proper non-singleton connected subset $B$. Choose $b_{1}, b_{2} \in B$ with $b_{1} \neq b_{2}$ and $x_{0} \in X \backslash B$. Let

$$
\mathcal{A}=\left\{A \in S(X): A=\left\{b_{1}\right\} \text { or } A \ni x_{0}\right\} \cup\{\emptyset\} .
$$

Then $\mathcal{A}$ satisfies conditions (a)-(c) in Lemma 1 , and $\left\{b_{2}\right\} \notin \mathcal{A}$. However, $\left\{b_{2}\right\} \in \operatorname{Lat}(\operatorname{Alg}(\mathcal{A}))$, which implies that $X$ is not s-reflexive. In fact, if $f \in$ $\operatorname{Alg}(\mathcal{A})$ and $b \in B$, from $\left\{b, x_{0}\right\} \in \mathcal{A}$ it follows that $f(b)=b$ or $f(b)=x_{0}$. Thus $B$ is the union of the disjoint closed sets $E=\{b \in B: f(b)=b\}$ and $K=\left\{b \in B: f(b)=x_{0}\right\}$. Trivially, $b_{1} \in E$, thus $E=B$ because $B$ is connected. In particular, $f\left(b_{2}\right)=b_{2}$. We are done.

THEOREM 4. A locally compact metric space is s-reflexive if and only if it is zero-dimensional (or, equivalently, if and only if it is strongly zerodimensional or hereditarily disconnected).

Proof. The equivalence of all the above conditions except s-reflexivity follows from [1, Theorem 6.2.9]. Since the s-reflexivity is topological and every locally compact metrizable space is completely metrizable, the result follows from Theorem 2 and Lemma 7.

In the following we construct a locally compact countable complete metric space which has a one-point extension that is not s-reflexive. We shall define a family of closed sets in the one-point extension space which satisfies all the conditions (a)-(d) in Lemma 1 and is not reflexive.

EXAMPLE 2. Let

$$
\begin{aligned}
& Y_{1}=\{(1 / n, m): n, m=1,2, \ldots\}, \\
& Y_{0}=\{(1 / n, 0): n=1,2, \ldots\} \cup\{(0,0)\} .
\end{aligned}
$$

As a subspace of $\mathbb{R}^{2}, Y=Y_{0} \cup Y_{1}$ is a locally compact countable complete space. Let $X=Y \cup\{p\}$, where $p$ is an element not in $Y$. Define a local base at $p$ as follows: for every map $g: \mathbb{N} \backslash D \rightarrow \mathbb{N} \cup\{0\}$, where $D$ is a finite subset of $\mathbb{N}$, let

$$
U(g)=\{p\} \cup\{(1 / n, m): n \in \mathbb{N} \backslash D, m>g(n)\} .
$$

Thus $(1 / n, m) \notin U(g)$ for any $n \in D$ and $m \in \mathbb{N} \cup\{0\}$. Note that $U(g) \cap$ $U(h)=U(\max \{g, h\})$, where $g: \mathbb{N} \backslash D_{1} \rightarrow \mathbb{N} \cup\{0\}, h: \mathbb{N} \backslash D_{2} \rightarrow \mathbb{N} \cup\{0\}$ and $\max \{g, h\}: \mathbb{N} \backslash\left(D_{1} \cup D_{2}\right) \rightarrow \mathbb{N} \cup\{0\}$ is defined by $\max \{g, h\}(n)=$ $\max \{f(n), g(n)\}$ for every $n \in \mathbb{N} \backslash\left(D_{1} \cup D_{2}\right)$. Thus all $U(g)$ 's form a local base at $p$. Assuming that $Y$ is an open subspace of $X$, we have thus defined a topology on $X$. 
We show that $X$ is not s-reflexive. Define a map $q: X \rightarrow Y_{0}$ by $q((1 / n, m))=(1 / n, 0), q(p)=q((0,0))=(0,0)$ for any $n \in \mathbb{N}$ and $m \in\{0\}$ $\cup \mathbb{N}$. Clearly, $q$ is continuous.

The family

$$
\mathcal{A}=\left\{q^{-1}(A): A \text { is closed in } Y_{0}\right\}
$$

satisfies conditions (a)-(c). In fact, (a) and (b) are clearly valid. To see that (c) is also satisfied, consider any family $\left\{A_{i}: i \in I\right\}$ of closed subsets of $Y_{0}$. Then $\operatorname{cl}\left(\bigcup_{i \in I} q^{-1}\left(A_{i}\right)\right)=\operatorname{cl}\left(q^{-1}\left(\bigcup_{i \in I} A_{i}\right)\right)=q^{-1}\left(\operatorname{cl}\left(\bigcup_{i \in I} A_{i}\right)\right)$. But $\mathcal{A}$ is not reflexive. In fact, $\{(0,0)\} \notin \mathcal{A}$. For any $f \in \operatorname{Alg}(\mathcal{A})$, as $\{(0,0), p\}=q^{-1}(\{(0,0)\})$ is in $\mathcal{A}$, we have $f((0,0))=(0,0)$ or $f((0,0))=p$. If the latter holds, then $p=\lim _{n \rightarrow \infty} f((1 / n, 0))$. By the definition of $\mathcal{A}$, $f((1 / n, 0)) \in q^{-1}(\{(1 / n, 0)\})$; set $f((1 / n, 0))=(1 / n, g(n)), n \in \mathbb{N}$. This yields a mapping $g: \mathbb{N} \rightarrow \mathbb{N} \cup\{0\}$. But then $f((1 / n, 0)) \notin U(g)$ for all $n \in \mathbb{N}$, which contradicts $p=\lim _{n \rightarrow \infty} f((1 / n, 0))$. This contradiction indicates $f((0,0))=(0,0)$, hence $\{(0,0)\} \in \operatorname{Lat}(\operatorname{Alg}(\mathcal{A}))$. Thus $\mathcal{A}$ is not reflexive.

Note that the only nonempty connected subsets of $X$ are singletons, so the family $\mathcal{A}$ constructed above also satisfies condition (d) in Lemma 1.

A closed subset $A$ of a space $X$ is called reflexive if $\{\emptyset, A, X\}$ is reflexive. Obviously both $\emptyset$ and $X$ are reflexive.

Lemma 8. A closed subset $A$ of a space $X$ is reflexive if and only if for each $B \in S(X)$ with $A \subset B \neq X$, there exists $f \in C(X)$ such that $f(A) \subseteq A$ but $f(B) \nsubseteq B$.

Proof. Assume that $A \neq \emptyset$ and $A \neq X$. The necessity is trivial. To show the sufficiency, suppose $B \in S(X)$ and $B \notin\{\emptyset, A, X\}$. If $A \not \subset B$, choose $a \in A \backslash B$ and define $f \in C(X)$ by $f(x)=a$ for all $x \in X$. Then $f(A) \subseteq A$ but $f(B) \nsubseteq B$. If $A \subset B$, it follows from the assumption that there exists $f \in C(X)$ such that $f(A) \subseteq A$ but $f(B) \nsubseteq \subseteq B$. Thus $\{\emptyset, A, X\}$ is reflexive.

Proposition 1. If each path-connected component of a Tikhonov space $X$ is dense in $X$, then every closed subset of $X$ is reflexive.

Proof. Let $A, B \in S(X)$ with $\emptyset \neq A \subset B \neq X$. Choose $a \in A$ and a continuous mapping $g: X \rightarrow[0,1]$ such that $g(x)=0$ for all $x \in A$ and $g(b)=1$ for some $b \in B$. Since the path component of $a$ intersects $X \backslash B$, there is a path $h:[0,1] \rightarrow X$ such that $h(0)=a$ and $h(1) \in X \backslash B$. Now $f=h \circ g \in C(X)$ and $f(A) \subseteq A$. However, $f(b)=h(1) \notin B$, so $f(B) \nsubseteq B$. By Lemma $8, A$ is reflexive.

Proposition 2. Every closed subset of a zero-dimensional space is reflexive. 
Proof. Let $X$ be a zero-dimensional space, and $A, B \in S(X)$ with $\emptyset \neq$ $A \subset B \neq X$. Choose $a \in A$ and $x_{0} \in X \backslash A$. There exists a clopen set $U \ni x_{0}$ such that $U \cap A=\emptyset$. Consider the mapping $f: X \rightarrow X$ defined by $f(x)=a$ if $x \notin U$ and $f(x)=x_{0}$ if $x \in U$. Then $f \in C(X)$ and $f(A) \subseteq A$ but $f(B) \nsubseteq B$.

Acknowledgements. The authors would like to express their sincere thanks to the referees for their valuable comments and suggestions. We also want to thank Prof. Chen Yixiang of Shanghai Teachers University for hosting a seminar in the Summer of 2002 which provided the two authors the chance to meet and initiate the current work.

\section{References}

[1] R. Engelking, General Topology, Polish Sci. Publ., Warszawa, 1977.

[2] P. R. Halmos, Ten problems in Hilbert space, Bull. Amer. Math. Soc. 76 (1970), 887-933.

[3] —, Reflexive lattices of subspaces, J. London Math. Soc. 4 (1971), 257-263.

[4] K. J. Harrison and W. E. Longstaff, Automorphic images of commutative subspace lattices, Proc. Amer. Math. Soc. 296 (1986), 217-228.

[5] W. E. Longstaff, Strongly reflexive subspace lattices, J. London Math. Soc. 11 (1975), 491-498.

[6] -, On lattices whose every realization on Hilbert space is reflexive, ibid. 37 (1988), 499-508.

[7] W. E. Longstaff and P. Oreste, On the ranks of single elements of reflexive operator algebras, Proc. Amer. Math. Soc. 125 (1997), 2875-2882.

[8] D. S. Zhao, On reflexive subobject lattices and reflexive endomorphism algebras, Comment. Math. Univ. Carolin. 44 (2003), 23-32.

Department of Mathematics

Shantou University

Shantou, Guangdong, 515063, P.R. China

E-mail: zqyang@stu.edu.cn
Mathematics and Mathematics Education National Institute of Education Nanyang Technological University 1 Nanyang Walk, Singapore 637616 E-mail: dongsheng.zhao@nie.edu.sg

Received 17 January 2005;

in revised form 19 July 2006 